\title{
The role of attention in binding visual features in working memory: evidence from cognitive ageing.
}

\author{
Louise A. Brown \\ The University of Edinburgh, Edinburgh, UK \\ James R. Brockmole \\ University of Notre Dame, Notre Dame, IN, USA
}

To cite:

Brown, L.A., \& Brockmole, J.R. (2010). The role of attention in binding visual features in working memory: evidence from cognitive ageing. The Quarterly Journal of Experimental Psychology, 63, 2067-2079. DOI:10.1080/17470211003721675 


\begin{abstract}
Two experiments were conducted to assess the costs of attentional load during a feature (colour-shape) binding task in younger and older adults. Experiment 1 showed that a demanding backwards counting task, which draws upon central executive/general attentional resources, reduced binding to a greater extent than individual feature memory, but the effect was no greater in older than younger adults. Experiment 2 showed that presenting memory items sequentially rather than simultaneously, such that items are required to be maintained while new representations are created, selectively affects binding performance in both age groups. Although this experiment exhibited an age-related binding deficit overall, both age groups were affected by the attention manipulation to an equal extent. While a role for attentional processes in colour-shape binding was apparent across both experiments, manipulations of attention exerted equal effects in both age groups. We therefore conclude that age-related binding deficits neither emerge nor are exacerbated under conditions of high attentional load. Implications for theories of visual working memory and cognitive ageing are discussed.
\end{abstract}




\section{Author Notes}

This research was funded by European Research Council grant 201312 awarded to J.R.B. who is also an Honorary Fellow at the University of Edinburgh. We thank Ian Deary, Alan Gow, and Paula Davies from the Lothian Birth Cohort Project, as well as the Lothian Birth Cohort 1936 Participants, for their assistance with this project. Correspondence should be addressed to Louise Brown, Department of Psychology, The University of Edinburgh, 7 George Square, Edinburgh, EH8 9JZ, louise.brown@ed.ac.uk; or to James Brockmole, University of Notre Dame, 118 Haggar Hall, Notre Dame, IN 46556, james.brockmole@nd.edu. 
The ability to remember visual material such as objects and faces over a short period of time decreases with age (see Reuter-Lorenz \& Sylvester, 2005, for a review). While several hypotheses have been offered for general working memory decline (e.g., Craik \& Byrd, 1982; Hasher \& Zacks, 1988; Salthouse, 1991), relatively little research has been specifically devoted to understanding age-related decline in visual working memory. This is surprising given that working memory deficits for visuo-spatial material have been shown to be more severe than those observed for verbal material (e.g., Jenkins, Myerson, Hale, \& Fry, 1999; Jenkins, Myerson, Joerding, \& Hale, 2000; Leonards, Ibanez, \& Giannakopoulos, 2002; Myerson, Hale, Rhee, \& Jenkins, 1999; but see Kemps \& Newson, 2006; Park, Lautenschlager, Hedden, Davidson, Smith, et al., 2002; Salthouse, 1995). One recently hypothesised source of visual working memory decline is an age-related impairment in the ability to maintain or 'bind' the correct associations between items of related information. Because objects are defined by different combinations of visual features such as colour, luminance, shape, size, orientation, and texture, one's ability to recall objects accurately depends upon retaining the associated features (e.g., that the car was blue and the lorry was red, or vice versa). Thus, an age-related binding deficit may explain the general performance decrements that have been observed in visual working memory across the adult lifespan.

However, evidence regarding the existence of an age-related binding deficit in visual working memory is mixed. There is some evidence for an age-related deficit in objectlocation binding (Cowan, Naveh-Benjamin, Kilb, \& Saults, 2006; Mitchell, Johnson, Raye, Mather, and D'Esposito, 2000), while binding of an object's surface features such as colour and shape thus far appears to be robust across the adult lifespan (Brockmole, Parra, Della Sala, \& Logie, 2008; Parra, Abrahams, Logie, \& Della Sala, 2009). Brockmole et al. (2008) argued that these latter findings pose a difficulty for the hypothesis that associative deficits lie at the heart of age-related decline in visual working memory. While age-related binding deficits may explain working memory problems when object-to-location binding is required, 
they may not account for other kinds of memory deficits. Instead, the binding deficit hypothesis may best explain age-related deficits in long-term memory which do appear to result, at least in part, from multiple types of binding failures (Chalfonte \& Johnson, 1996; Naveh-Benjamin, 2000; Naveh-Benjamin, Guez, Kilb, \& Reedy, 2004; Naveh-Benjamin, Hussain, Guez, \& Bar-On, 2003; Old \& Naveh-Benjamin, 2008). However, Brockmole et al. (2008) noted that the possibility of specific age-related binding deficits between features in visual working memory cannot yet be completely discounted, because they may exist under certain circumstances.

In this paper we consider whether age-related binding deficits emerge under conditions of high attentional load. It has been consistently reported that decreased central executive functioning plays an important role in general age-related cognitive decline (e.g., Craik, 1983; Craik \& Byrd, 1982; Hasher \& Zacks, 1988). For example, greater age-related memory deficits tend to be found in novel tasks which draw heavily upon cognitive resources, and in tasks which require active manipulation of information (e.g., see Craik \& Anderson, 1999; Kester, Benjamin, Castel, \& Craik, 2002). These deficits are linked to structural (Haug \& Eggers, 1991; Murphy, DeCarli, McIntosh, Daly, Mentis, et al., 1996; West, 1996) and functional (Chao \& Knight, 1997; Murphy et al., 1996) changes to the frontal lobes which are associated with central executive functioning (Baddeley, 1986; Collette \& Van der Linden, 2002; Miller \& Cohen, 2001). Furthermore, older adults have been shown to exhibit deficits in executive behaviours associated specifically with dorsolateral prefrontal cortex activity (MacPherson, Phillips, \& Della Sala, 2002), while fronto-temporal circuitry has recently been suggested to underlie age-related cognitive decline (Brickman, Zimmerman, Paul, Grieve, Tate, et al., 2006).

If the maintenance of feature conjunctions requires general attentional (central executive) resources, memory binding errors may result when attention is taxed during the retention interval. Given the evidence reviewed above, this effect may be more pronounced in 
older adults. To date, however, the role of attention in feature binding has only been addressed with younger adults, and the results have been mixed. Some evidence suggests that attention is needed to maintain proper associations between features (e.g., Wheeler \& Treisman, 2002). For example, the requirement to remember a string of digits, monosyllabic words, or pure tones disrupts visual-spatial, verbal-spatial, and object-feature binding (Elsley \& Parmentier, 2009a, 2009b). Some other attentionally demanding tasks such as multiple object tracking have also been shown to affect working memory for concurrently maintained bound features to a greater extent than for individual features (Fougnie \& Marois, 2009). These results suggest that binding across working memory components, as well as within the visual working memory component alone, involves the recruitment of general attentional resources. Additionally, feature bindings appear to be more fragile than individual feature representations in visual working memory. For example, binding performance is selectively affected by sequential versus simultaneous presentation of memory items, suggesting that inter-item interference and overwriting by newly processed and stored items result in fragile bindings (Allen, Baddeley, \& Hitch, 2006; see also Alvarez \& Thompson, 2009; Logie, Brockmole, \& Vandenbroucke, 2009). In contrast, other studies have supported the conclusion that feature conjunctions can be maintained without need for continued attention. Gajewski and Brockmole (2006) showed that, in the face of attentional distraction, observers remember either all of an object's features or none at all. In addition, Allen et al. (2006) and Johnson, Hollingworth, and Luck (2008) have shown that a variety of dual tasks fail to differentially reduce memory for bound objects compared to individual features.

The relationship between attention and binding clearly remains unresolved, but this inconsistency does not preclude the hypothesis that age-related binding deficits arise when individuals are placed under high task loads. The studies below sampled two tasks that have supported both points of view on the role of attention in binding, allowing us to investigate whether age-related changes to the attention system moderate feature binding. We examined 
whether age-related binding deficits a) emerge in tasks that have not exhibited deficits in younger adults, and b) are exacerbated in tasks that have demonstrated a role of attention in binding. Experiment 1 considered the efficacy of binding when individuals must engage in a concurrent task requiring attentional resources, while Experiment 2 investigated how memory is affected by the requirement to create new bindings while maintaining previously encountered ones.

\section{Experiment 1}

The purpose of Experiment 1 was to determine whether a concurrent attentionally demanding task differentially affects binding abilities in younger and older adults. The experiment was based on the procedures and results obtained by Allen et al. (2006, Exp. 4), who required young participants to remember coloured shapes while counting backwards by threes from a randomly selected three-digit number. These researchers found that this attentionally demanding task yielded no greater deficit in memory for conjunctions of features compared to individual features, providing support for the argument that binding does not rely upon central executive resources.

In the present experiment performance of younger and older adults was assessed under conditions in which they were required to remember colours, shapes, or colour-shape bindings while either simply repeating a two digit number (control condition) or counting backwards by threes from a two-digit number (counting condition). This backward counting task was required throughout the encoding, maintenance, and retrieval phases of the object memory task in order to maximize the potential cost of the secondary task on binding performance. On the basis of Allen et al.'s findings, we predicted that younger adults would suffer no specific binding deficit due to the backwards counting task, but the most important question was whether this same result would hold for older adults. On the basis of Brockmole et al.'s (2008) results, in which no attention manipulations were carried out and no specific- 
age related binding deficits were observed, one could predict no differential effect of the counting task on binding performance. However, if, unlike younger adults, older adults rely more heavily upon general attentional resources for colour-shape binding, then the counting task would be expected to affect older adults' binding performance to a greater extent than younger adults.

\section{Method}

Participants. In total there were 48 participants. There were 24 younger adults from the student population of The University of Edinburgh, aged between 18-22 years $(M=$ $18.96, S D=1.00)$, with a mean number of years of education of $13.96(S D=1.83)$ and a mean verbal IQ, as predicted by the National Adult Reading Test (NART), of 104.67 (SD = 5.22). These participants received either course credit or an honorarium. The older adults comprised 24 volunteers randomly selected from the Lothian Birth Cohort 1936 (LBC1936; see Deary, Gow, Taylor, Corley, Brett, et al., 2007), for whom there was a range of demographic data already available. Due to their membership of the Cohort, the older adults were all either 72 or 73 years of age $(M=72.21, S D=0.42)$. Their mean number of years of education was $13.67(S D=3.61)$ and their NART-predicted verbal IQ was $110.67(S D=$ 11.44). Predicted verbal IQ was slightly higher in older than younger adults, $t(46)=-2.34, p$ $<.05$. Note, however, that this difference is in the opposite direction of the age-based memory effects reported below, and is likely to reflect that the younger adults are yet to develop their verbal knowledge fully. Sex distribution was the same for both age groups (8 males and 16 females). Additionally, the older participants were screened for unhealthy cognitive decline using the Mini-Mental State Examination (MMSE; Folstein, Folstein, \& McHugh, 1975), for which their mean score was 29.17 ( $S D=1.05)$. Participants reported no significant memory problems and normal or corrected-to-normal vision. 
Stimuli and Apparatus. Simuli consisted of coloured shapes presented on a grey background. Memory displays were composed of three objects, each of which was created by combining one of six colours (red, yellow, blue, green, cyan, and purple) and one of six shapes (circle, triangle, diamond, heart, arrow, and cross) randomly without replacement. Test arrays were composed of a single item which differed across three visual task conditions (described below). In the colour memory task the test item was a non-canonically shaped 'blob' presented in one of the six colours listed above. In the shape memory task, the test item was one of the six shapes listed above drawn with a black outline but filled in grey to match the background. In the feature binding (conjunction) memory task, each test item was a coloured shape, as in the memory array. Stimuli were presented on a 17" CRT monitor with a screen refresh rate of $85 \mathrm{~Hz}$. Each stimulus measured approximately $1.6 \mathrm{~cm}^{2}$, and viewing distance was not constrained.

Design and procedure. Prior to the main task younger adults completed the NART to obtain an estimate of verbal IQ (these data were already available for the older adults). The main experiment was then administered. The procedure is illustrated in Figure 1.

(Figure 1 about here)

Participants initiated each trial by pressing the space bar. A randomly-generated twodigit number (between 20-99) was then displayed in the centre of the screen for $2 \mathrm{~s}$. Immediately, participants said this number out loud and either continued to repeat this number out loud at their own pace (control task) or carried out backwards counting by threes from this number out loud (counting task). Both the control and counting tasks served to suppress articulatory rehearsal. The experimenter recorded the number of repetitions in the control task and the number of articulated calculations in the counting task, with the latter 
data allowing scoring of the total number of calculations made and the correct number of calculations made.

After the two-digit number had disappeared from the screen a central fixation cross was presented for $1000 \mathrm{~ms}$ (which remained until the end of the trial) after which an array of three coloured shapes were displayed in a row in three fixed locations above the cross for $900 \mathrm{~ms}$. After a delay of $1000 \mathrm{~ms}$, a test item appeared below one of the original memory items (the position of the probe was randomly selected). Participants responded 'yes' or 'no', by pressing the ' $\mathrm{z}$ ' or ' $\mathrm{m}$ ' keys on a keyboard, to indicate whether or not the colour, shape, or colour-shape conjunction depicted in the test item had appeared in the memory array, irrespective of location. Incorrect colour-shape conjunctions were created by errantly combining a colour and shape that were present in the initial memory array (cf. Wheeler \& Triesman, 2002). Participants continued to repeat or count until this key-press response had been made.

The experiment was divided into six blocks of trials which orthogonally combined the three memory and the two secondary tasks. Control and counting blocks were yoked such that participants completed all three blocks involving repetition followed by all three blocks involving counting, or vice versa. The order of memory tasks was also counterbalanced within the constraint that each participant completed the memory tasks in the same order for each concurrent task. This resulted in 12 orders of administration which were repeated twice within each age group. There were six practice trials followed by 36 experimental trials in each block, with half of the test items present and half absent. Trial order was randomised across participants.

\section{Results and Discussion}

Neither the mean number of repetitions nor the mean number of correct calculations per trial varied across age groups or memory tasks (all $p$ 's $>.31$ ). Overall, the mean number of 
correct calculations per trial was 2.48 ( $S D=.69$; see Table 1 for scores in each condition), while the mean number of repetitions per trial was $5.21(S D=1.37)$.

Response accuracy was expressed as A' with a 'hit' defined as the correct detection of change. The mean A' scores broken down by each factor in the experimental design are displayed in Figure 2.

(Figure 2 about here)

These data were analysed using a 2 (age) x 2 (concurrent task) x 3 (visual memory task) mixed-model analysis of variance (ANOVA). Main effects of age group, $F(1,46)=6.75$, $M S E=.021, p<.02$, concurrent task type, $F(1,46)=46.76, M S E=.009, p<.001$, and visual memory task type, $F(2,92)=54.62, M S E=.006, p<.001$, were observed. Overall, visual memory was superior in younger adults $(M=.88)$ than older adults $(M=.83)$ and better in the control task $(M=.89)$ than the counting task $(M=.82)$. Visual memory for colour $(M=$ .91) was superior to that for shape $(M=.86)$ and bindings $(M=.79)$, a finding that is typical in the literature (Allen et al., 2006; Brockmole et al., 2008; Johnson et al., 2008; Wheeler \& Treisman, 2002). The only interaction was between concurrent task type and visual memory task type, $F(2,92)=18.95, M S E=.005, p<.001($ all other $p$ 's $>.13)$.

With no interactions involving age reaching statistical significance, planned comparisons (paired t-tests) that collapsed across the age factor were conducted to explore the effect of concurrent task on memory task type. Within the control task, performance was better in the colour than in the shape block, $t(47)=8.08, p<.001$, while there was no difference between performance in the shape and binding blocks, $t(47)<1$. This indicates that memory for bindings was no worse than memory for the least well remembered feature (i.e., shape memory, which established the lower bound on performance). This outcome is 
one hallmark of successful and efficient binding ${ }^{1}$ (Luck \& Vogel, 1997). In contrast, within the counting task, there were differences between the colour and shape blocks, $t(47)=2.47, p$ $<.02$, as well as between the shape and binding blocks, $t(47)=6.02, p<.001$. This indicates that binding memory was poorer than memory for individual features and shows that the counting task disrupted binding ability.

Because we emphasized accuracy rather than speed, an analysis of response times (RT) provides limited probative value with respect to the specific experimental hypotheses; however, it is apparent from the RT data that no speed accuracy trade-offs were evident. The control task $(M=1182 \mathrm{~ms})$ yielded faster RTs than the counting task $(M=1464 \mathrm{~ms}), F(1,46)$ $=40.67, M S E=141289, p<.001$, and the colour memory condition $(M=1199 \mathrm{~ms})$ was responded to faster than both the shape $(M=1374 \mathrm{~ms})$ and binding $(M=1394 \mathrm{~ms})$ conditions, $F(2,92)=18.44, M S E=59942, p<.001$. Also, younger adults $(M=1099 \mathrm{~ms})$ exhibited faster RTs than the older adults $(M=1546 \mathrm{~ms}), F(1,46)=22.06, M S E=651237, p$ $<.001$. No significant interactions were observed involving RT.

Although backwards counting disrupted memory for bindings more than memory for individual features, from Experiment 1 it is clear that older adults did not exhibit a specific age-related colour-shape binding deficit under circumstances where an attentionally demanding concurrent task was required during the encoding, retention, and recall of visual objects. This result is consistent with previous failures to find age-related binding deficits in visual working memory (Brockmole et al., 2008; Parra et al., 2009). The fact that the binding performance of older adults did not suffer disproportionately compared to younger adults

\footnotetext{
${ }^{1}$ Because binding performance was numerically lower than shape memory performance in older adults, we carried out a t-test to confirm that there was no reliable difference between shape and binding memory performance in older adults, $t(23)=1.57, p=.13$. It could be argued that the lack of age-related binding deficit is due to a type II error. Note, however, that the sample size was larger here than in previous reports (e.g., Brockmole et al., 2008; Parra et al., 2009) that also failed to find age-related binding deficits, and was equivalent to that used in Experiment 2 of the current paper which does observe a reliable age-related binding deficit.
} 
from the requirement to carry out a concurrent task which draws upon central executive resources, suggests that older adults do not rely upon central executive resources for binding any more than do younger adults.

Before concluding our discussion of Experiment 1, we note that the overall binding deficit caused by the counting task, that was independent of age, runs contrary to the findings of Allen et al. (2006), who used this same manipulation to conclude that binding does not require general attentional resources (see also, Gajewski \& Brockmole, 2006; Luck \& Vogel, 1997; Vogel, Woodman, \& Luck, 2001, for similar conclusions from other tasks). While a complete account of this disparity falls outside the scope of this report, we suggest that subtle differences between our methods may have resulted in our study being more sensitive to a specific decrease in binding task performance. Due to our inclusion of older participants, we used a three item instead of four item memory array in order to lower overall task difficulty. As a result of their higher memory loads, Allen et al. reported lower baseline levels of memory performance as well as greater overall decrements due to the counting task. Consequently, they reported a hit rate at chance level (0.49) for their binding memory task when accompanied by counting. Thus, a floor effect may have obscured a binding deficit in the face of the counting task. Additionally, Allen et al. only required backwards counting to continue until the test array was presented whereas we were strict in ensuring that counting was performed until a response was made. It is possible that higher task demands during the retrieval phase of the task also exacerbated the effect of the task on bindings. We suggest that either or both of these factors may have resulted in increased sensitivity in the current study. Nevertheless, the main findings of Experiment 1 regarding age are clear; a concurrent task, which had the power to cause general binding deficits, exerted an equivalent effect on both younger and older adults. 


\section{Experiment 2}

The purpose of Experiment 2 was to investigate whether age exacerbates the propensity for feature conjunctions to dissociate as new objects are processed. In everyday life, retention intervals do not span blank displays. Instead, processing of additional or newly encountered objects must take place. According to feature integration theory, however, the binding of features into integrated object percepts requires attention (e.g., Treisman, 1986, 1999; Treisman \& Gelade, 1980). Because older adults have been shown to suffer from decreased frontal lobe function and attentional resources (e.g., Brickman et al., 2006; Chao \& Knight, 1997; Hasher, Stoltzfus, Zacks, \& Rypma, 1991; Hasher \& Zacks, 1988; Haug \& Eggers, 1991; MacPherson et al., 2002; Murphy et al., 1996; West, 1996), we could predict a greater binding deficit for older than younger adults when both creating and maintaining object representations. Specifically, bindings may be more susceptible to interference from sequential (one item at a time) versus simultaneous presentation.

Experiment 2 was based on a recent experiment with young adults reported by Allen et al. (2006; Exp. 5) which investigated whether the binding of new object representations disrupts the maintenance of previously encoded objects by causing their feature bindings to dissociate. Using a change detection task, they examined memory for features and their conjunctions in situations where all objects in the memory set were simultaneously presented and where the objects appeared one at a time. Memory performance was found to be poorer in the sequential presentation condition, with memory for conjunctions showing a greater decrement than that for single features. In Experiment 2, we considered whether this deficit is more pronounced in older adults. 


\section{Method}

Participants. In total there were 48 participants. There were 24 younger adults from the student population of The University of Edinburgh, aged between 18-24 years $(M=$ $20.33, S D=1.93)$, with a mean number of years of education of $15.57(S D=2.09)$ and a mean NART-predicted verbal IQ of $106.04(S D=7.86)$. These participants received either course credit or an honorarium. As in Experiment 1, the older adults comprised 24 volunteers randomly selected from LBC1936 who did not participate in Experiment 1 (Deary et al. 2007). Their mean age was $72.33(S D=0.48)$ and their mean number of years of education was $13.81(S D=3.09)$. Their NART-predicted verbal IQ was $115.63(S D=6.28)$ and, as in Experiment 1, this was slightly higher than in younger adults, $t(46)=-4.67, p<.001$. The older participants were screened for unhealthy cognitive decline using the MMSE, and their mean score was $29.04(S D=1.23)$. Sex distribution was the same for both age groups (10 males and 14 females). Participants reported no significant memory problems and normal or corrected-to-normal vision.

Stimuli. The stimuli were the same as in Experiment 1.

Design and Procedure. The experimental procedure was the same as in Experiment 1 except that stimulus display times were altered to accommodate the presentation manipulation. In the simultaneous condition, all aspects of the procedure were the same as the control condition of Experiment 1, except that the memory array was displayed for $1500 \mathrm{~ms}$. In the sequential presentation condition, the procedure was the same as the simultaneous condition except that the memory array items were presented one at a time, from the left position through to the right, for $500 \mathrm{~ms}$ each (ISI was $0 \mathrm{~ms}$ ). Aside from these alterations, all other aspects of the experimental design and procedure were the same as the control condition of Experiment 1. 


\section{Results and Discussion}

The rate at which numbers were vocalised in the articulatory suppression task did not differ across age groups or memory tasks (all $p$ 's $>.15$ ). Overall, the mean number of repetitions per trial was $6.15(S D=1.54)$, a similar rate to that observed in Experiment 1 , when taking into account the slightly longer trial duration in Experiment 2.

As in Experiment 1, response accuracy was expressed as A' with a 'hit' defined as the correct detection of change. The mean A' scores are displayed in Figure 3.

(Figure 3 about here)

These data were analysed using a 2 (age group) x 2 (presentation format) x 3 (memory task) mixed ANOVA. Main effects of age group $F(1,46)=38.96, M S E=.012, p<.001$, presentation format, $F(1,46)=5.16, M S E=.004, p<.03$, and memory task type, $F(2,92)=$ 110.32, MSE $=.006, p<.001$, were observed. Overall, visual memory was superior in younger adults $(M=.93)$ than older adults $(M=.85)$ and when tested with simultaneous presentation $(M=.90)$ compared to sequential presentation $(M=.88)$. Visual memory for colour $(M=.96)$ was superior to that for shape $(M=.91)$ which, in turn, was superior to bindings $(M=.80)$. There was, however, an interaction between the effects of age group and memory task type, $F(2,92)=12.23, M S E=.006, p<.001$, and also between presentation format and memory task type, $F(2,92)=15.81, M S E=.004, p<.001$. Remaining interactions were not significant (all $p$ 's $>.24)$.

To follow up on the interaction between age group and memory task, performance on each memory task (collapsing across presentation format) was analysed within each age group individually (see Figure 4). 
Within the younger age group, there was a main effect of memory task, $F(2,46)=$ 41.47, $M S E=.002, p<.001$, and planned comparisons (paired t-tests) revealed that performance for colour was better than for shape, $t(23)=5.35, p<.001$, and that performance for shape was better than for binding, $t(23)=5.54, p<.001$. Within the older age group, there was also a main effect of memory task, $F(2,46)=69.70, M S E=.004, p<.001$, again with performance for colour better than for shape, $t(23)=5.86, p<.001$, and performance for shape better than for binding, $t(23)=7.13, p<.001$. However, the effect of memory task was larger for the older adults (estimated effect size of block type in younger adults, $\eta_{\mathrm{p}}{ }^{2}=.64$, and in older adults, $\eta_{\mathrm{p}}{ }^{2}=.75$ ). Moreover, this disparity is most evident in the binding condition where the difference between the binding task and the single feature tasks was greater among older adults. Due to the lack of a three-way interaction involving presentation format, we can conclude that an equivalent age-related binding deficit was observed in both the simultaneous and sequential conditions.

To follow up on the interaction between presentation format and memory task, memory task performance (collapsing across age) was assessed within each presentation format individually. Differences between memory task performances were evident in both the simultaneous, $F(2,94)=37.06, M S E=.004, p<.001$, and the sequential conditions, $F(2,94)$ $=80.27, M S E=.007, p<.001$. However, as confirmed by planned comparisons (paired $\mathrm{t}-$ tests), the differences were greater in the sequential condition. While performance was the same between the simultaneous and sequential formats for colour, $t(47)=-.47, p=n s$, and only slightly different for shape, $t(47)=-2.31, p<.03$, performance was much better in the simultaneous than the sequential format for binding, $t(47)=3.90, p<.001$. Furthermore, the estimated effect size of memory task was much larger in the sequential condition, $\eta_{\mathrm{p}}{ }^{2}=.63$, than in the simultaneous condition, $\eta_{\mathrm{p}}^{2}=.44$. This finding indicates a specific deficit for binding with sequential presentation. Due to the lack of a three-way interaction involving 
age, we can conclude that the deficit arising from sequential presentation was equivalent for younger and older adults.

Finally, we note that no speed-accuracy trade-offs were present in the data. The simultaneous task $(M=1240 \mathrm{~ms})$ was responded to slightly quicker than the sequential task $(M=1296 \mathrm{~ms}), F(1,46)=5.25, M S E=43492, p<.03$. The colour memory condition $(M=$ $1101 \mathrm{~ms})$ was responded to faster than the shape condition $(M=1308 \mathrm{~ms})$, while shape RTs were faster than binding RTs $(M=1396 \mathrm{~ms}), F(2,92)=62.19, M S E=35461, p<.001$. Younger adults $(M=951 \mathrm{~ms})$ exhibited faster RTs than the older adults $(M=1585 \mathrm{~ms})$, $F(1,46)=75.48, M S E=383678, p<.001$. Importantly, however, there were no significant interactions involving RT.

As in Experiment 1, Experiment 2 has demonstrated general binding deficits in the face of an attentional manipulation, in this case the need to create bindings while others are maintained. Although sequential presentation selectively disrupted binding performance across both age groups (cf. Allen et al., 2006; Alvarez \& Thompson, 2009; Logie et al., 2009), older adults were no more affected by sequential presentation than were the younger adults. Curiously, however, an age-related binding deficit was observed independently of the attentional manipulation. Of the seven experiments we know of that have been conducted to search for such an effect (three reported by Brockmole et al., 2008, two reported by Parra et al., 2009, and the two reported here), this is the first experiment to obtain such a finding. While the preponderance of the evidence indicates that, under conditions of little attentional load, older adults do not display age-related binding deficits (aside from those involving explicit recall of location), this experiment keeps the issue open for further scrutiny. Here, however, in combination with the results from Experiment 1, we may confidently conclude that attentional load does not lead to or exacerbate an age-related binding deficit for object surface features in visual working memory. 


\section{General Discussion}

Previous research has considered whether age-related declines in visual working memory can be explained by associative deficits which result in a decreased ability to generate and store bound object representations. This hypothesis arose, in part, from demonstrations of such deficits in long-term memory (Naveh-Benjamin, 2000) as well as some evidence indicating that older adults have more difficulty remembering object-location bindings in working memory (Cowan et al., 2006; Mitchell, Johnson, Raye, Mather, et al., 2000). Despite the promise of this hypothesis, evidence for age-related binding deficits in visual working memory for visual features such as colour and shape has been elusive (Brockmole et al., 2008; Parra, et al., 2009). The present study investigated the effect of attentional load on visual working memory for individual and bound features in order to establish whether or not an age-related binding deficit arises when executive resources are taxed. Because attention resources become more difficult to manage as we age, we hypothesised that binding deficits could emerge under conditions of high attention load.

In a single-probe change-detection paradigm, Experiment 1 showed that a demanding concurrent task selectively affected binding performance in both age groups, but that the older adults exhibited no greater deficit than did the younger adults. In Experiment 2, to-beremembered items were presented either simultaneously or sequentially in order to assess the effect that concurrently maintaining and creating bindings exerts on memory. The results supported previous evidence that bound object representations are fragile in visual working memory (Allen et al., 2006; Alvarez \& Thompson, 2009; Logie et al., 2009) as they are susceptible to interference from the encoding, processing, and/or maintenance of other items. However, older adults were no more affected by sequential presentation than were younger adults. Taken together, the results of these two experiments suggest that the attention deficits associated with aging (see Craik, 1983; Craik \& Byrd, 1982; Hasher \& Zacks, 1988) neither bring about, nor exacerbate, binding deficits in visual working memory. These results 
complement and extend the conclusions of Cowan et al. (2006) who, in the context of objectlocation binding deficits in working memory, showed that older adults exhibit a deficit over and above that of an attentional one, and argued that the binding deficit is related to a lack of processing robustness and a degradation in memory performance itself. Furthermore, Mitchell, Johnson, Raye, and D'Esposito (2000) provided fMRI evidence that hippocampal functional deficits are related to binding impairments in working memory. Finally, in the domain of long-term memory, Naveh-Benjamin, Guez, and Shulman (2004) showed that the age-related binding deficit is not explained by attentional deficits. It therefore appears that, where age-related binding deficits exist, they appear not to be related to attentional limitations.

As noted earlier, the results of Experiment 2 offer the first suggestion of an age-related binding deficit for the surface features of objects. While it is not clear why this result was obtained, we suggest that it may be related to stimulus presentation time. This could relate to the claims of Cowan et al. (2006), that processing robustness may be a factor affecting binding performance in older adults. The one systematic difference between the control conditions of Experiment 1 and Experiment 2 concerns the duration of stimulus presentation (900 ms in Experiment 1 and $1500 \mathrm{~ms}$ in Experiment 2), which suggests that encoding time is an important factor in binding. Indeed, converging evidence for this is available in the literature. Tasks which tend to show an involvement of attention in binding have generally used longer stimulus presentation times than those that have failed to find such an effect. As exemplified in dual-task studies, Allen et al. (2006) presented their to-be-remembered stimuli for $250 \mathrm{~ms}$ and Johnson et al. (2008) presented their stimuli for $500 \mathrm{~ms}$. In contrast, we presented our stimuli in Experiment 1 for 900 ms, and Elsley and Parmentier (2009a, 2009b) presented their stimuli for $2000 \mathrm{~ms}$. The first two studies found no relationship between attention and binding (presentation times $<500 \mathrm{~ms}$ ) while the latter two did so (presentation times $>900 \mathrm{~ms}$ ). It therefore seems clear that future work with both younger and older adults 
would benefit from the direct assessment of the role of presentation time on binding memory performance.

Focussing on the results within younger adults, our results speak to the general role of attention in feature binding. Our evidence supports recent claims in the literature that attention plays a role in memory for bound object representations (Elsley \& Parmentier, 2009a, 2009b; Fougnie \& Marois, 2009; Wheeler \& Treisman, 2002). Although other attempts to find attention-related binding deficits have been unsuccesful (e.g., Allen et al., 2006; Gajewski \& Brockmole, 2006; Johnson, et al., 2008), the format of the attentional tasks and differences in memory task demands or sensitivity may account for the disparities. We suggest that dual tasks which make sufficient demands upon general attention, and that accompany memory tasks in which baseline performance is sufficiently high, should disrupt binding memory to a greater extent than individual feature memory (i.e., Experiment 1 of the current paper; Elsley \& Parmentier 2009b). Theoretically, the impact of general attentional demands on feature binding would be predicted by Baddeley's (2000) working memory model, in which the episodic buffer component is responsible for binding features together via the general attentional resources of the central executive. Because Allen et al. (2006) found no dual task decrements for binding, they concluded that within-modality binding may be carried out relatively automatically at the level of the working memory subsystem and that the episodic buffer may only be required for cross-modal binding. The results of Experiment 1 of the current paper, however, suggest that binding of features within the visuo-spatial subsystem do draw upon central executive resources in the fashion predicted by the Baddeley (2000) model, rather than being carried out more automatically, and therefore the episodic buffer may indeed be required to account for this.

In conclusion, the results of the current study indicate that age-related binding deficits may contribute to a general decline in visual working memory abilities in some circumstances. That said, while such deficits are robustly observed in long-term episodic 
memory, they have proven to be much more difficult to observe in visual working memory (e.g., Experiment 1 of the current paper; Brockmole et al., 2008; Parra et al., 2009), although a variety of other task demands and situations have yet to be considered. What is clear from these findings is that age-related declines in attention resources do not increase the likelihood that binding will fail. Instead, manipulations of attention affect binding equally for both younger and older adults. In the context of Baddeley's (2000) working memory model, this last observation suggests that visual feature bindings do draw upon central executive resources, perhaps in the episodic buffer component, and are not automatically stored within the visuospatial subsystems as has been suggested previously.

\section{References}

Allen, R. J., Baddeley, A. D., \& Hitch, G. J. (2006). Is the binding of visual features in working memory resource-demanding? Journal of Experimental Psychology: General, 135, 298-313.

Alvarez, G. A., \& Thompson, T. W. (2009). Overwriting and rebinding: Why feature-switch detection tasks underestimate the binding capacity of visual working memory. Visual Cognition, 17, 141-159.

Baddeley, A. D. (1986). Working memory. Oxford, UK: Oxford University Press.

Baddeley, A. D. (2000). The episodic buffer: A new component of working memory? Trends in Cognitive Sciences, 4, 417-423.

Brickman, A. M., Zimmerman, M. E., Paul, R. H., Grieve, S. M., Tate, D. F., Cohen, R. A., et al. (2006). Regional white matter and neuropsychological functioning across the adult lifespan. Biological Psychiatry, 60, 444-453. 
Brockmole, J. R., Parra, M. A., Della Sala, S., \& Logie, R. H. (2008). Do binding deficits account for age-related decline in visual working memory? Psychonomic Bulletin \& Review, 15, 543-547.

Chalfonte, B. L., \& Johnson, M. K. (1996). Feature memory and binding in young and older adults. Memory \& Cognition, 24, 403-416.

Chao, L. L., \& Knight, R. T. (1997). Prefrontal deficits in attention and inhibitory control with aging. Cerebral Cortex, 7, 63-69.

Collette, F., \& Van der Linden, M. (2002). Brain imaging of the central executive component of working memory. Neuroscience and Biobehavioral Reviews, 26, 105-125.

Cowan, N., Naveh-Benjamin, M., Kilb, A., \& Saults, J. S. (2006). Life-span development of visual working memory: When is feature-binding difficult? Developmental Psychology, $42,1089-1102$.

Craik, F. I. M. (1983). On the transfer of information from temporary to permanent memory. Philosophical Transactions of the Royal Society of London, B302, 341-359.

Craik, F. I. M., \& Anderson, N. D. (1999). Applying cognitive research to problems of aging. In D. Gopher, \& A. Koriat (Eds.), Attention and Performance XVII (pp. 583-615). Cambridge, MA: MIT Press.

Craik, F. I. M., \& Byrd, M. (1982). Aging and cognitive deficits: The role of attentional resources. In F. I. M. Craik \& S. Trehub (Eds.), Aging and cognitive processes (pp. 191-211). New York: Plenum Press.

Deary, I. J., Gow, A. J., Taylor, M. D., Corley, J., Brett, C., Wilson, V., et al. (2007). The Lothian Birth Cohort 1936: a study to examine influences on cognitive ageing from age 11 to age 70 and beyond. BMC Geriatrics, 7; 28.

Elsley, J., \& Parmentier, F. B. R. (2009a). Does binding in visuo-spatial working memory require attention? As cited in J. Elsley, \& F. B. R. Parmentier (2009), Is verbal-spatial 
binding in working memory impaired by a concurrent memory load? Quarterly Journal of Experimental Psychology, 62, 1696-1705.

Elsley, J., \& Parmentier, F. B. R. (2009b). Is verbal-spatial binding in working memory impaired by a concurrent memory load? Quarterly Journal of Experimental Psychology, 62, 1696-1705.

Folstein, M. F., Folstein, S. E., \& McHugh, P. R. (1975). "Mini-Mental State": A practical method for grading the cognitive state of patients for the clinician. Journal of Psychiatric Research, 12, 189-198.

Fougnie, D., \& Marois, R. (2009). Attentive tracking disrupts feature binding in visual working memory. Visual Cognition, 17, 48-66.

Gajewski, D. A., \& Brockmole, J. R. (2006). Feature bindings endure without attention: Evidence from an explicit recall task. Psychonomic Bulletin \& Review, 13, 581-587.

Hasher, L., Stoltzfus, E. R., Zacks, R. T., \& Rypma, B. (1991). Age and inhibition. Journal of Experimental Psychology: Learning, Memory, and Cognition, 17, 163-169.

Hasher, L., \& Zacks, R. T. (1988). Working memory, comprehension, and aging: a review and a new view. In G. G. Bower (Ed.), The psychology of learning and motivation, Vol. 22 (pp. 193-225). San Diego, CA: Academic Press.

Haug, H., \& Eggers, R. (1991). Morphometry of the human cortex cerbri and corpus striatum during aging. Neurobiology of Aging, 12, 336-338.

Jenkins, L., Myerson, J., Hale, S., \& Fry, A. F. (1999). Individual and developmental differences in working memory across the life span. Psychonomic Bulletin \& Review, 6, $28-40$.

Jenkins, L., Myerson, J., Joerding, J. A., \& Hale, S. (2000). Converging evidence that visuospatial cognition is more age-sensitive than verbal cognition. Psychology and Aging, 15, 157-175. 
Johnson, J. S., Hollingworth, A., \& Luck, S. J. (2008). The role of attention in the maintenance of feature bindings in visual short-term memory. Journal of Experimental Psychology: Human Perception and Performance, 34, 41-55.

Kemps, E., \& Newson, R. (2006). Comparison of adult age differences in verbal and visuospatial memory: The importance of 'pure', parallel and validated measures. Journal of Clinical and Experimental Neuropsychology, 28, 341-356.

Kester, J. D., Benjamin, A. S., Castel, A. D., \& Craik, F. I. M. (2002). Memory in elderly people. In A. D. Baddeley, M. D. Kopelman, \& B. A. Wilson (Eds.), Handbook of memory disorders ( $2^{\text {nd }}$ ed., pp. 543-567). Chichester, UK: Wiley.

Leonards, U., Ibanez, V., Giannakopoulos, P. (2002). The role of stimulus type in age-related changes of visual working memory. Experimental Brain Research, 146, 172-183.

Logie, R. H., Brockmole, J. R., \& Vandenbroucke, A. R. E. (2009). Bound feature combinations in visual short-term memory are fragile but influence long-term learning. Visual Cognition, 17, 160-179.

Luck, S. J., \& Vogel, E. K. (1997). The capacity of visual working memory for features and conjunctions. Nature, 390, 279-281.

MacPherson, S. E., Phillips. L. H., \& Della Sala, S. (2002). Age, executive function, and social decision making: A dorsolateral prefrontal theory of cognitive aging. Psychology and Aging, 17, 598-609.

Miller, E. K., \& Cohen, J. D. (2001). An integrative theory of prefrontal cortex function. Annual Review of Neuroscience, 24, 167-202.

Mitchell, K. J., Johnson, M. K., Raye, C. L., \& D'Esposito, M. (2000). fMRI evidence of age-related hippocampal dysfunction in feature binding in working memory. Cognitive Brain Research, 10, 197-206. 
Mitchell, K. J., Johnson, M. K., Raye, C. L., Mather, M., \& D’Esposito, M. (2000). Aging and reflective processes of working memory: Binding and test load deficits. Psychology and Aging, 15, 527-541.

Murphy, D. G. M., DeCarli, C., McIntosh, A. R., Daly, E., Mentis, M. J., Pietrini, P., et al. (1996). Sex differences in human brain morphometry and metabolism: An in vivo quantitative magnetic resonance imaging and positron emission tomography study on the effect of aging. Archives of General Psychiatry, 53, 585-594.

Myerson, J., Hale, S., Rhee, S. H., \& Jenkins, L. (1999). Selective interference with verbal and spatial working memory in young and older adults. Journal of Gerontology: Psychological Sciences, 54, 161-164.

Naveh-Benjamin, M. (2000). Adult age-differences in memory performance: Tests of an associative deficict hypothesis. Journal of Experimental Psychology: Learning, Memory, and Cognition, 26, 1170-1187.

Naveh-Benjamin, M., Guez, J., Kilb, A., \& Reedy, S. (2004). The associative memory deficit of older adults: Further support using face-name associations. Psychology \& Aging, 19, $541-546$.

Naveh-Benjamin, M., Guez., J., \& Shulman, S. (2004). Older adults' associative deficit in episodic memory: Assessing the role of decline in attentional resources. Psychonomic Bulletin \& Review, 11, 1067-1073.

Naveh-Benjamin, M., Hussain, Z., Guez, J., \& Bar-On, M. (2003). Adult age-differences in episodic memory: Further support for an associative deficit hypothesis. Journal of Experimental Psychology: Learning, Memory, and Cognition, 29, 826-837.

Old, S. R., \& Naveh-Benjamin, M. (2008). Memory for people and their actions: Further evidence for an age-related associative deficit. Psychology and Aging, 23, 467-472. 
Park, D. C., Lautenschlager, G., Hedden, T., Davidson, N. S., Smith, A.D., \& Smith, P. K. (2002). Models of visuospatial and verbal memory across the adult lifespan. Psychology and Aging, 17, 299-320.

Parra, M. A., Abrahams, S., Logie, R. H., \& Della Sala, S. (2009). Age and binding withindimension features in visual short-term memory. Neuroscience Letters, 449, 1-5.

Reuter-Lorenz, P. A., \& Sylvester, C. C. (2005). The cognitive neuroscience of working memory and aging. In R. Cabeza, L. Nyberg, \& D. Park (Eds.), The Cognitive Neuroscience of Aging: Linking Cognitive and Cerebral Aging (pp. 186-217). New York: Oxford University Press.

Salthouse, T. A. (1991). Theoretical perspectives on cognitive aging. Hillside, NJ: Erlbaum.

Salthouse, T. A. (1995). Differential age-related influences on memory for verbal-symbolic information and visual-spatial information. Journal of Gerontology: Psychological Sciences, 50, 193-201.

Treisman, A. (1986). Features and objects in visual processing. Scientific American, 225, 114-125.

Treisman, A. (1999). Feature bindings, attention, and object perception. In G. W. Humphreys, J. Duncan, \& A. Treisman (Eds.), Attention, space, and action: Studies in cognitive neuroscience (pp. 91-111). New York: Oxford University Press.

Treisman, A. M., \& Gelade, G. (1980). Feature-integration theory of attention. Cognitive Psychology, 12, 97-136.

Vogel, E. K., Woodman, G. F., \& Luck, S. J. (2001). Storage of features, conjunctions, and objects in visual working memory. Journal of Experimental Psychology: Human Perception and Performance, 27, 92-114.

West, R. L. (1996). An application of prefrontal cortex function theory to cognitive aging. Psychological Bulletin, 120, 272-292. 
BINDING IN VISUAL WORKING MEMORY 28

Wheeler, M. E., \& Treisman, A. M. (2002). Binding in short-term visual memory. Journal of Experimental Psychology: General, 131, 48-64. 
Table 1

Performance of the backwards counting task of Experiment 1 (mean number of correct calculations with standard deviations) for each age group across each condition.

\begin{tabular}{|l|c|c|c|}
\hline & Colour & Shape & Binding \\
\hline Younger & $2.63(0.64)$ & $2.62(0.64)$ & $2.67(0.65)$ \\
\hline Older & $2.30(0.76)$ & $2.31(0.78)$ & $2.37(0.74)$ \\
\hline
\end{tabular}




\section{Figure Captions}

Figure 1: Experiment 1 procedure. Note that display items are not drawn to scale and that colours are depicted by different fill effects.

Figure 2: Mean A' values (and standard errors) for younger and older adults across each condition in Experiment 1.

Figure 3: Mean A' values (and standard errors) for younger and older adults across each condition in Experiment 2.

Figure 4: Mean A' values (and standard errors) for younger and older adults in each memory task of Experiment 2, collapsed across presentation format. 
Figure 1

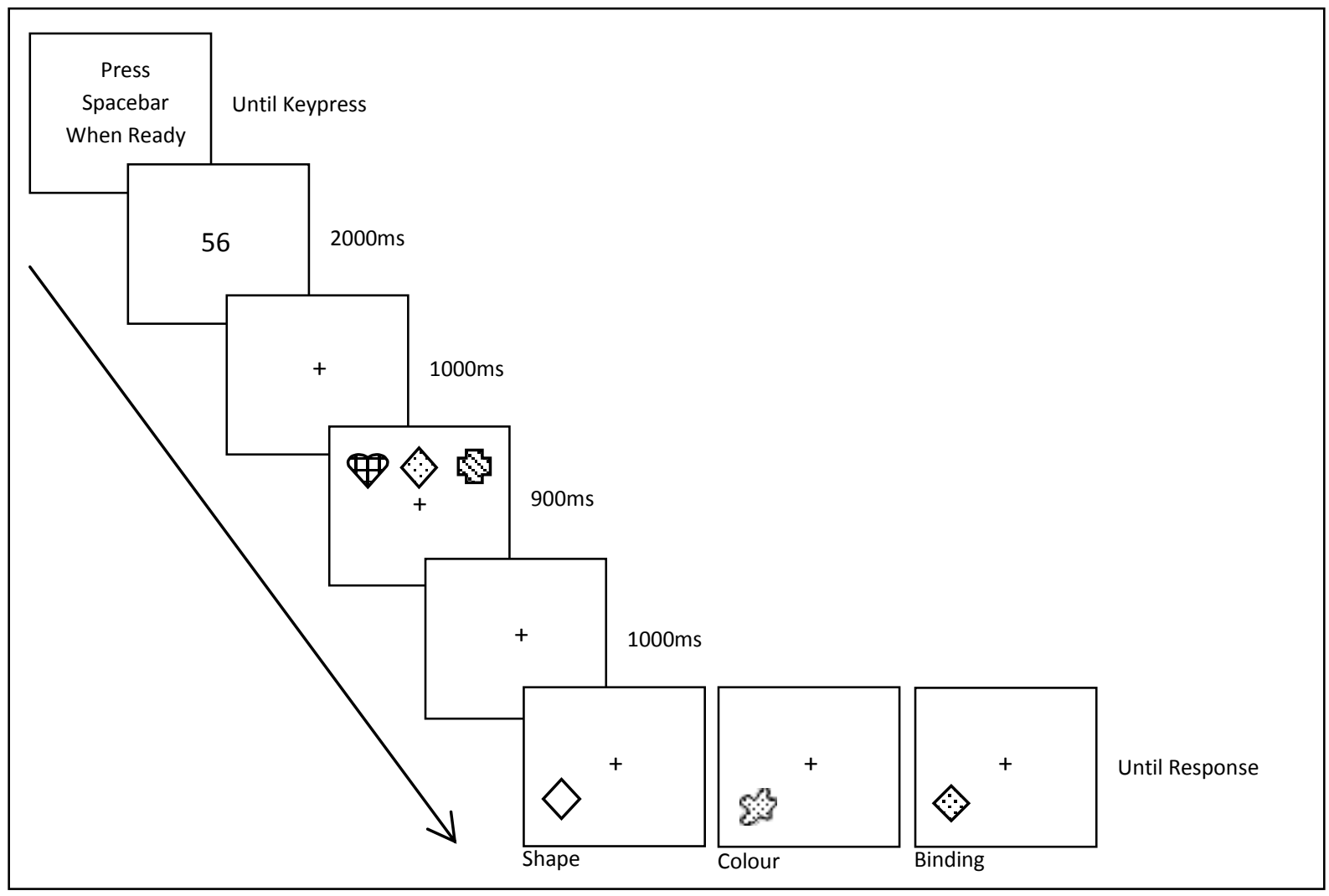


Figure 2

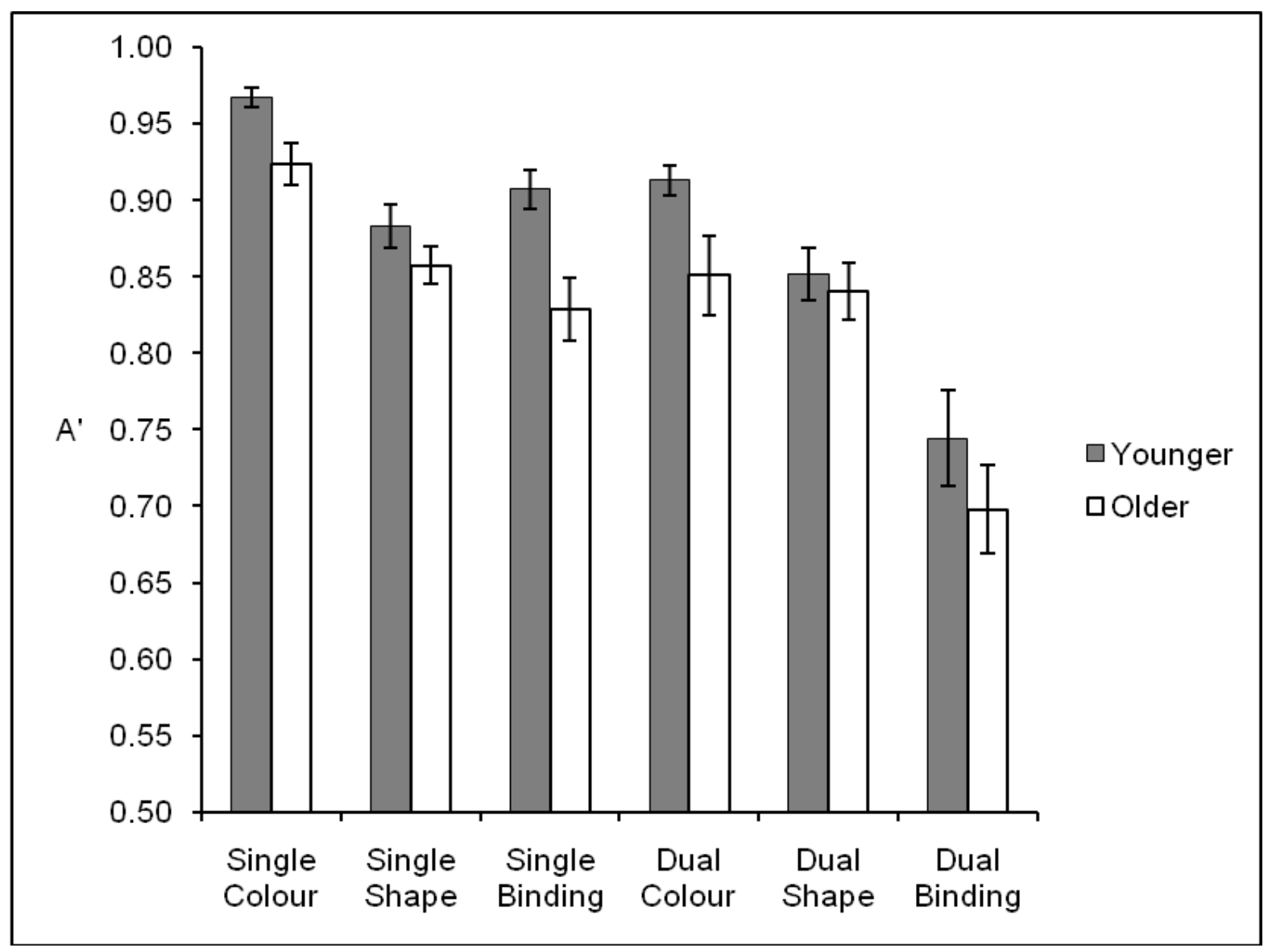


Figure 3

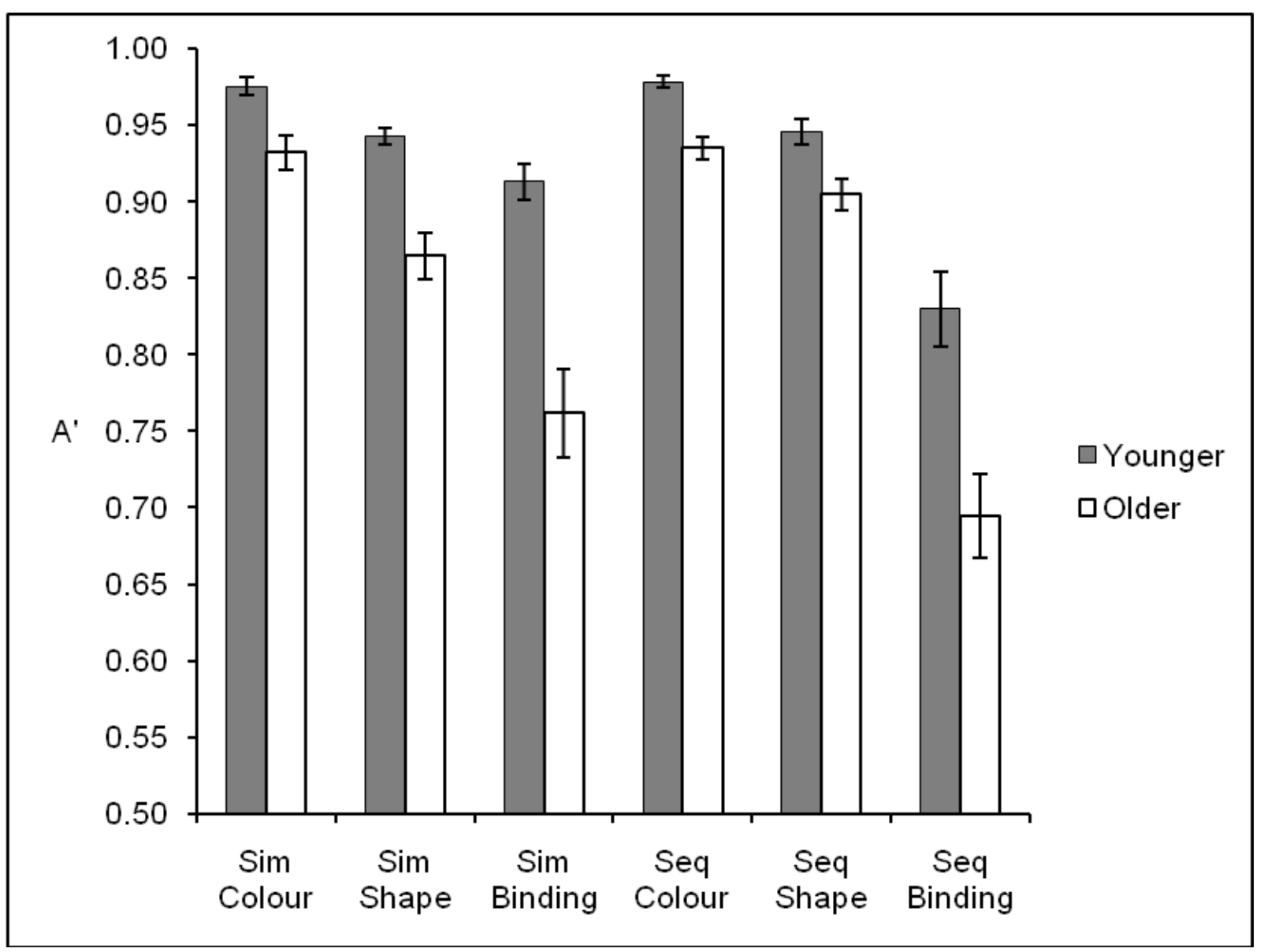


Figure 4

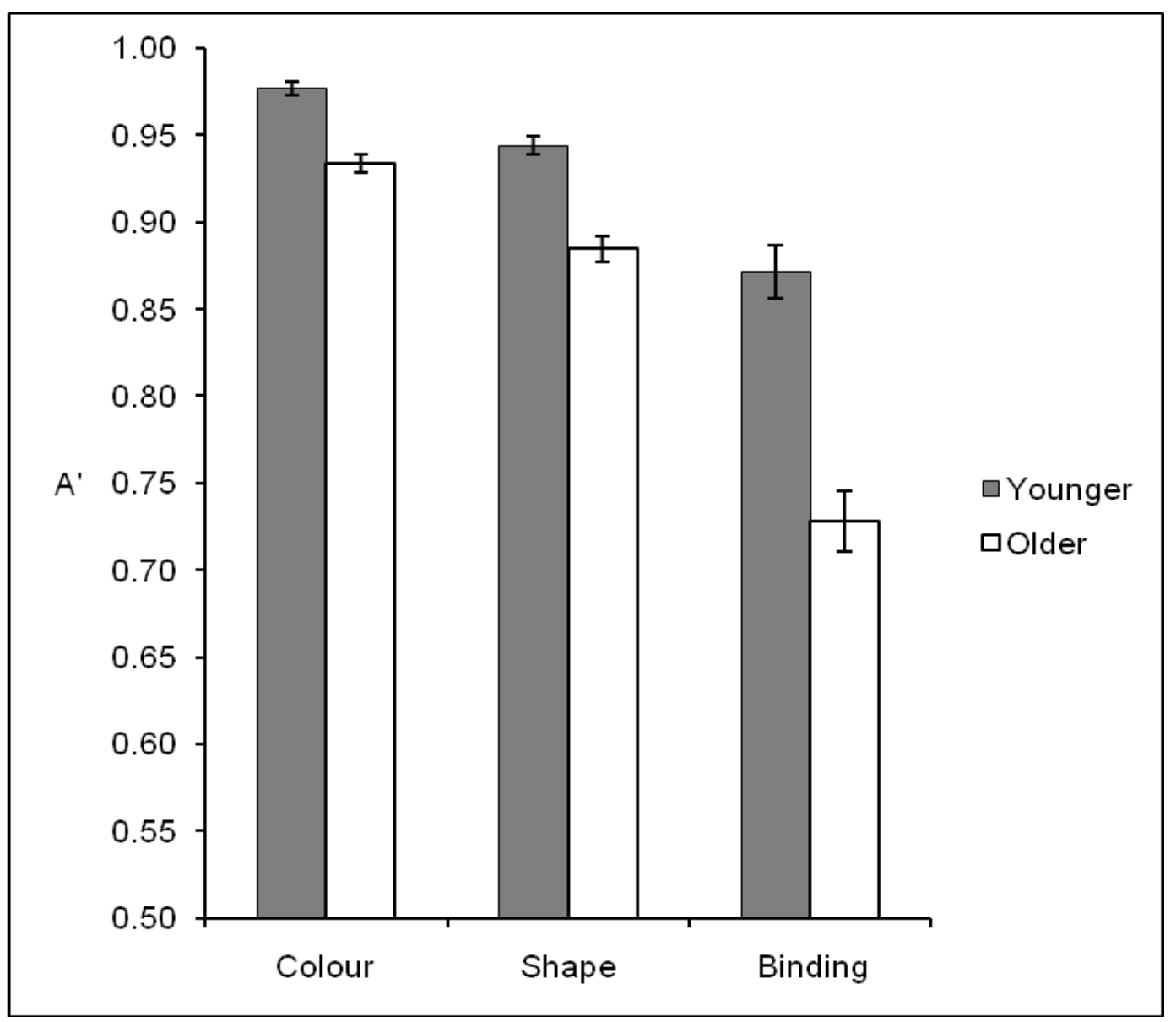

